\title{
$O$ cálculo do poder das lentes intra-oculares e o Orbscan-II. Parte 1: 0 poder óptico da córnea normal
}

\author{
Powercalculation of intraocularlenses and the Orbscan-II. \\ Part 1: The power of the normal cornea
}

\author{
Érika C. Canarim de Oliveira ${ }^{1}$ \\ Carlos G. Arce 2 \\ Mauro Campos ${ }^{3}$ \\ Paulo Schor ${ }^{4}$
}

\begin{tabular}{|c|}
\hline RESUMO \\
\hline $\begin{array}{l}\text { Objetivo: Avaliar o poder dióptrico da porção central da córnea normal. } \\
\text { Métodos: Análise retrospectiva da topografia por varredura de fenda de luz } \\
\text { e disco de Plácido em } 30 \text { olhos normais. Resultados: Os poderes médios } \\
\text { ceratométrico, anterior, da espessura e total; ópticos ceratométrico e total; } \\
\text { axiais ceratométrico e total; tangencial total, equivalente e a soma do poder } \\
\text { médio de ambas superfícies foram semelhantes }(p \geq 0,8361) \text { nas quatroáreas } \\
\text { centrais analisadas }(0,04,1,3 \text { e } 5 \mathrm{~mm} \text { de diâmetro). O poder médio posterior } \\
\text { diminuiu de }-6,62 \mathrm{D} \text { até }-6,35 \mathrm{D}(p=0,0030) \text {. A espessura média aumentou } \\
\text { de } 524 \mu \mathrm{m} \text { até } 554 \mu \mathrm{m}(p=0,0231) \text {, representando } 0,13 \mathrm{D} \text { do poder total da } \\
\text { córnea. Os poderes óptico ( } p \leq 0,0167) \text {, axial }(p \leq 0,0099) \text { e médio ( } p \leq 0,0030) \\
\text { ceratométricos foram aproximadamente } 1,50 \mathrm{D} \text { mais positivos que os } \\
\text { respectivos poderes totais. O poder médio total foi igual ao poder equiva- } \\
\text { lente ( } p \geq 0,4907) \text { e à soma do poder de ambas superfícies ( } p \geq 0,3868) \text {. O } \\
\text { poder médio anterior foi ao redor de } 7,5 \text { vezes maior que o poder médio } \\
\text { posterior. Conclusões: } O \text { poder real da córnea calculado usando ambas } \\
\text { superfícies, sua espessura e os índices de refração fisiológicos é menor que } \\
\text { o poder convencionalmente aceito e determinado pela medição da curva- } \\
\text { tura da superfície anterior e o índice de refração ceratométrico da córnea } \\
\text { ( } F<K) \text {. O poder da superfície posterior e a paquimetria variam conformeo } \\
\text { tamanho da área central estudada. O poder da superfície anterior e o poder } \\
\text { total se mantêm os mesmos. Os componentes do poder total da córnea e } \\
\text { a paquimetria parecem ser independentes entre si. }\end{array}$ \\
\hline
\end{tabular}

Descritores: Córnea/fisiologia, Topografia da córnea/estatística \& dados numéricos, Refração ocular, Lentes intra-oculares

\section{INTRODUÇ̃̃̃O}

mento de Oftalmologia da Universidade Federal de São Paulo - UNIFESP e Mestranda do Departamento de Oftalmologia, Otorrinolaringologia e Cabeça e Pescoço da Faculdade de Medicina de Ribeirão Preto - USP. ${ }^{2}$ Pesquisador Associado do Departamento de Oftalmologia da Universidade Federal de São Paulo - UNIFESP.

${ }_{3}^{3}$ Chefe do Setor de Cirurgia Refrativa do Departamento de Oftalmologia da Universidade Federal de São Paulo - UNIFESP.

${ }^{4}$ Professor Afiliado do Departamento de Oftalmologia da Universidade Federal de São Paulo - UNIFESP.

Endereço para correspondência: Carlos G. Arce

Rua Borges Lagoa, 368 - São Paulo (SP) CEP 04038-000 E-mail: cgarce@mpc.com.br

Recebido para publicação em 09.09.2002 Aceito para publicação em 20.10.2002

Nota Editorial: Pela análise deste trabalho e por sua anuência na divulgação desta nota, agradecemos aos Drs. Paulo André Polisuk e Maurício Brik.

\begin{tabular}{l}
\hline INTRODUÇ̃̃o \\
\hline O poder óptico da córnea $(K)$, em dioptrias, é convencionalmente deter- \\
minado pela medição do raio de curvatura $(r)$ da sua superfície anterior pela \\
ceratometria manual e, mais recentemente, pela computadorizada e pelo \\
sistema de análise topográfico ${ }^{(1-2)}$. A sua aceitação como um dado válido, \\
se fundamenta na fórmula da "lente fina" $\left(K=n_{2}-n_{l} / r\right)$, onde $n_{1}$ é o índice \\
de refração do ar e vale 1, e $n_{2}$ é o índice de refração ceratométrico (IRC) ou \\
índice de refração corrigido da córnea. Este IRC vale $1,3375^{(3)}$, embora \\
alguns aparelhos usam também 1,3313 ou $1,336^{(2-4)}$. O IRC é um valor \\
arbitrariamente assumido a partir do poder óptico $(+45 \mathrm{D})$ e do raio de \\
curvatura anterior $(7,5 \mathrm{~mm})$ da córnea no modelo de olho proposto por \\
Gullstrand há mais de 100 anos ${ }^{(3-6)}$. O IRC pressupõe uma superfície anterior \\
da córnea com um poder 10 vezes maior que o da superfície posterior ${ }^{(7)}$.
\end{tabular}


Para se calcular o poder refrativo real da córnea é preciso considerar que este sistema é composto de duas superfícies ópticas (faces anterior e posterior) separadas por uma distância entre elas (espessura) e de três meios ópticos (ar, córnea e humor aquoso) cada um com um índice de refração diferente (Figura 1). Enquanto que a espessura da córnea pode ser determinada pela paquimetria óptica ou pela ultra-sônica ${ }^{(8)}$, a limitação técnica de medir sua superfície posterior sempre dificultou a confirmação do valor de $K$, aferido nos equipamentos, como seu poder real ${ }^{(2,4)}$. Por outro lado, pela videoceratoscopia por varredura de fenda de luz combinada com a reflexão do disco de Plácido, milhares de pontos da córnea e da câmara anterior são localizados tridimensionalmente. O Orbscan-II, (Bausch \& Lomb-Orbtek Inc, Salt Lake City, Utah) originalmente lançado como um paquímetro computadorizado, usa diversas metodologias de cálculo para transformar esta informação em mapas topográficos de elevação, de poder ou paquimétricos, entre outros, não só da superfície anterior da córnea, mas também da sua superfície posterior, da sua espessura e da câmara anterior ${ }^{(7,9-12)}$.

Os mapas de elevação comparam ambas superfícies da córnea com as suas melhores esferas possíveis, ("best fit sphere"), distribuindo o que estiver desigual, tanto acima (áreas de cores mais quentes) quanto abaixo (áreas de cores mais frias) delas. A diferença de altura é expressa em $\mathrm{mm}$ de raio da esfera calculada pelo método do mínimo quadrado ("least square") de uma área, de diâmetro predeterminado, centralizada em um ponto de referencia (o eixo visual, o ápice ou o centro geográfico da córnea). O mapa paquimétrico mostra a diferença em elevação entre as superfícies anterior e posterior da córnea. Como esta medida é 5\% a $8 \%$ superior à paquimetria ultra-sônica, o Orbscan-II corrige esta informação mostrando o equivalente acústico da espessura da córnea ${ }^{(8-9,13-14)}$.

Dependendo do índice de refração usado ou da porção analisada da córnea, os diversos mapas de poder são subdivididos em ceratométrico, anterior, posterior, total e da espessura. Para os mapas de poder ceratométrico, o Orbscan-II usa automaticamente o IRC de 1,3375. Para os mapas de poder anterior, posterior e total da córnea usa a variação fisiológica dos índices de refração do ar $(1,0)$, da córnea $(1,376)$ e do humor aquoso

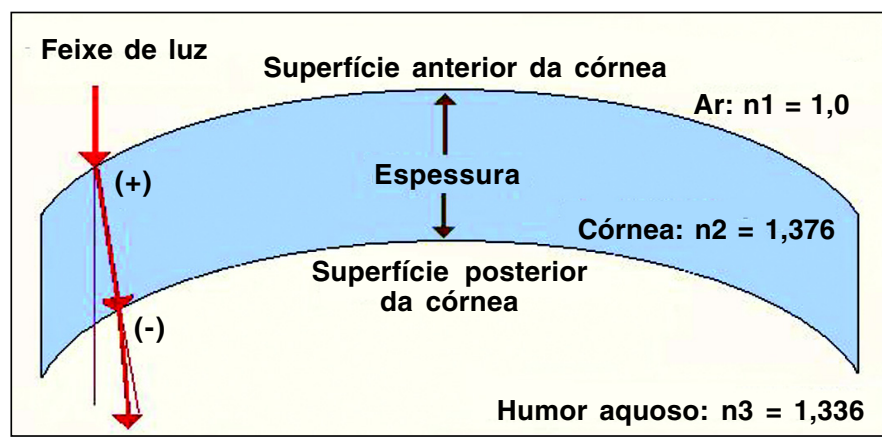

Figura 1 - Sistema óptico esquemático da córnea onde $n 1$ é o índice de refração do ar, n2 é o índice de refração do estroma da córnea e n3 é o índice de refração do humor aquoso
(1,336). O mapa de poder da espessura ("thickness power map") representa a contribuição, no poder total, da espessura da córnea medida perpendicularmente à superfície anterior.

Os mapas de poder são também classificados segundo sua forma de cálculo. Os mapas de poder médio ("mean power maps") representam o equivalente esférico, isto é, a média aritmética da curvatura, em dioptrias, de cada ponto nas duas superfícies da córnea, em todas as direções (meridianos). O mapa de poder médio total é a soma dos mapas de poder médio anterior, posterior e da espessura. Os mapas de poder óptico ("optical power maps") são uma representação da capacidade de focalização da córnea e são também denominados de poder refrativo ou de Snell. Os mapas de poder astigmático mostram o astigmatismo ponto por ponto da córnea. Os mapas de poder axial computam os raios de curvatura da superfície da córnea, em planos ou eixos individuais, centralizados na linha de visão. Os mapas de poder tangencial são um melhoramento dos mapas de poder axial e consideram um círculo tangencial a cada ponto analisado. Os mapas de poder óptico total, axial total e tangencial total representam a soma do poder das superfícies anterior e posterior da córnea, desconsiderando a espessura.

\section{OBJETIVOS}

O presente estudo tem por objetivo avaliar o poder da porção central da córnea normal, a partir das medidas fornecidas pela topografia por varredura de fenda de luz e disco de Plácido. Perguntas sobre os melhores parâmetros para uso nas fórmulas de cálculo de poder das lentes intra-oculares (LIOs), mapa a usar, área a analisar, ou valor óptico da espessura da córnea, deverão ser respondidas a partir do alicerce deste trabalho, com o intuito dirigido a resolver a questão de se o Orbscan-II é útil como ferramenta para minimizar os erros refracionais pós-facectomia nos pacientes submetidos a cirurgia refrativa da córnea.

\section{MÉTODOS}

Foi analisada a video-ceratoscopia por varredura de fenda de luz e disco de Plácido com o Orbscan-II de 30 olhos normais, de pacientes diferentes, selecionados pela ordem de arquivo no equipamento. A refração e a lateralidade do olho examinado não foram levadas em conta. Os mapas estudados foram os seguintes: de poder médio ceratométrico, médio anterior, médio posterior, médio da espessura e médio total, de poder óptico ceratométrico e óptico total, de poder axial ceratométrico e axial total, de poder tangencial total e o mapa paquimétrico. Considerou-se a média do poder da córnea e da paquimetria de áreas com $0,04,1,0,3,0$ e $5,0 \mathrm{~mm}( \pm 0,02 \mathrm{~mm}) \mathrm{de}$ diâmetro centralizadas na linha de visão, utilizando o recurso de "análise estatística de área" ("software" versão 3.00D) do equipamento (Figura 2). Uma análise preliminar parcial, utilizando o poder da córnea nas "zonas estatísticas" padrão (3,0 e 


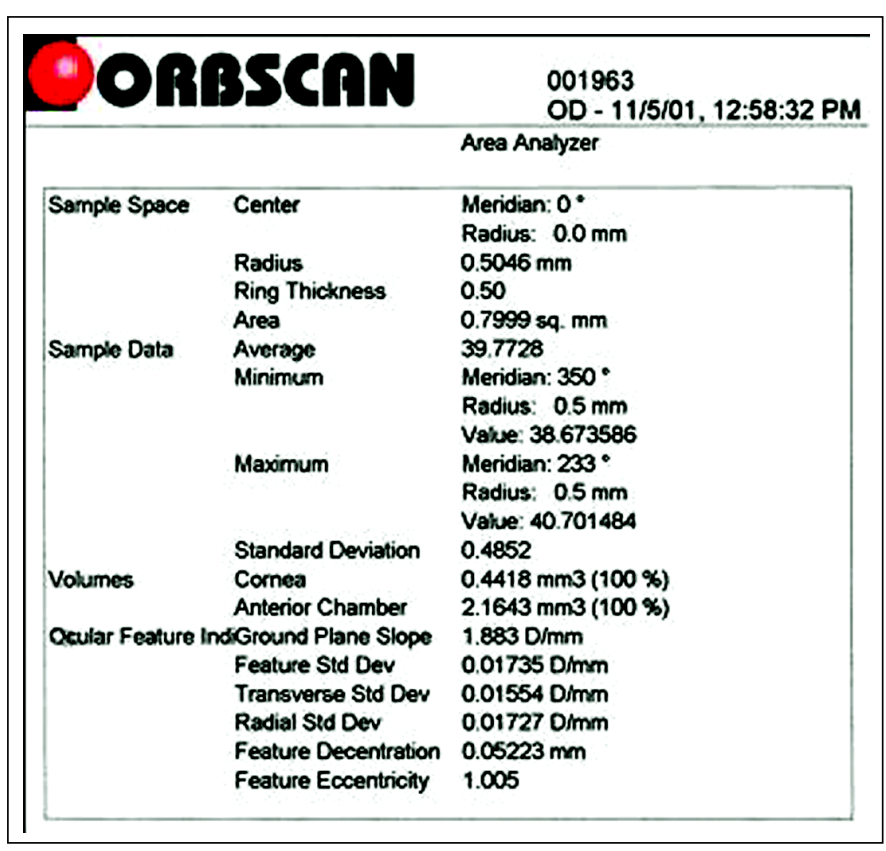

Figura 2 - Exemplo da janela da análise estatística de área mostrando o raio do círculo analisado, a sua área, o poder mínimo, o máximo, o médio e o desvio padrão

$5,0 \mathrm{~mm}$ ) e a paquimetria média da área central padrão de $2,0 \mathrm{~mm}$ (figura 3) foi realizada com o intuito de avaliar estes recursos.

$O$ poder equivalente da córnea foi calculado pela fórmula da "lente grossa": $F=F a+F p-(d / n) F a{ }_{*} F p$, ${ }^{(15)}$ onde $F$ é o poder equivalente da córnea em dioptrias, $F a$ é o poder médio em dioptrias da superfície anterior da córnea; $F p$ é o poder médio em dioptrias da superfície posterior da córnea; $d$ é a espessura média em metros, $n$ é o índice de refração 1,376 do estroma da córnea, e $d / n$ é a "espessura reduzida" da córnea. Como alguns autores desconsideram o poder derivado da espessura por ser pequeno ${ }^{(2,4)}$, calculamos também, para fins comparativos, a soma dos mapas de poder médio anterior e posterior (poder médio da soma $\mathrm{A} / \mathrm{P}$ ).

Foram utilizados o teste " $t$ " com presunção de variâncias equivalentes e médias iguais, o teste Anova com fator único, o fator de correlação de Pearson $(R)$ e o cálculo do $R^{2}$ do programa MS-Excel para um $p<0,025$.

\section{RESULTADOS}

O Orbscan-II repete nos mapas de elevação anterior, de poder óptico anterior e de poder médio ceratométrico os mesmos valores para as áreas centrais padrão de 3,0 e 5,0 mm pelo recurso da "zona estatística". O mesmo foi observado para os mapas de poder médio anterior e de poder axial anterior. $\mathrm{O}$ poder da superfície posterior, independente do tipo de mapa, foi sempre o mesmo.

A Tabela 1 apresenta as médias ( \pm desvio padrão) dos dados obtidos com o recurso da "análise estatística de área", do poder médio da soma $\mathrm{A} / \mathrm{P}$ e do poder equivalente. Nos mapas de poder médio ceratométrico, médio anterior e médio total, óptico ceratométrico e óptico total, axial ceratométrico, axial total e tangencial total, as médias foram semelhantes $(p \geq 0,8361)$ nas quatro áreas centrais estudadas. Os únicos mapas de poder com variação significativa $(p \leq 0,003)$ foram os de poder médio posterior, que diminuíram conforme aumentou a área analisada (média de $-6,62 \mathrm{D}$ para os $0,04 \mathrm{~mm}$ centrais até $-6,35 \mathrm{D}$ para os $5 \mathrm{~mm}$ centrais).

As médias dos mapas de poder óptico ceratométrico (entre $+44,33 \mathrm{e}+44,80 \mathrm{D})$ foram as mais próximas a $+45 \mathrm{D}$, o poder aceito convencionalmente para a córnea padrão. Por outro lado, as médias dos mapas de poder médio anterior foram sempre muito superiores a +45 D (entre +48,97 e +48,67 D). As médias dos mapas de poder óptico ceratométrico $(p \leq 0,0167)$, axial ceratométrico, $(p \leq 0,0099)$, e médio ceratométrico ( $p \leq 0,0030)$, também foram maiores que as médias dos respectivos mapas de poder total, nas quatro áreas centrais estudadas. Em nenhum olho, nas quatro áreas analisadas, a diferença entre o poder médio ceratométrico e o poder médio total foi menor a $+0,75 \mathrm{D}$, e apenas em dois, na área $\leq 0,04 \mathrm{~mm}$, foi maior a $+2,0 \mathrm{D}$ (Tabela 2).

As médias dos mapas de poder médio total resultaram iguais às do poder equivalente da córnea (diferença entre $0,005 \mathrm{D}$ e $0,011 \mathrm{D}, p \geq 0,4907$, Pearson $\cong 1,0)$, e às do poder médio da soma $\mathrm{A} / \mathrm{P}(p \geq 0,3868$, Pearson $\cong 1,0$, tabela 2$)$. A espessura média da córnea aumentou de $524 \mu \mathrm{m}$ no centro até $554 \mu \mathrm{m}$ na área de $5 \mathrm{~mm}(p \leq 0,0231)$, porém a sua contribuição no poder total da córnea foi sempre a mesma (ao redor de $+0,136 \mathrm{D}$, $p \geq 0,8678$; Tabela 1).

O poder médio da superfície anterior foi, em média, entre 7,4 e 7,7 vezes maior que o poder médio da superfície posterior. A correlação entre os poderes médios anterior e posterior não foi muito boa nas áreas menores, embora tenha aumentado progressivamente nas áreas maiores. (Pearson $=-0,451$ no centro até Pearson $=-0,865$ na área com $5 \mathrm{~mm}$, tabela 3 ). Esta correlação não pareceu ser linear, quadrática, cúbica, de quarto grau nem logarítmica, (Figura 2 e Tabela 4). O poder médio atribuído à espessura foi, em média, entre 358 e 366 vezes menor que o poder médio da superfície anterior e entre 47 e 49 vezes menor que o poder médio da superfície posterior. As correlações, nestes dois últimos casos (Tabela 3 ), e aquela entre a paquimetria e os três componentes do poder óptico da córnea (Tabela 4) também não foram boas.

\section{DISCUSSÃO}

A enorme quantidade de informação, proporcionada pela topografia por varredura de fenda de luz e disco de Plácido, cria, paradoxalmente, certa confusão sobre o valor que seria mais apropriado aplicar nos cálculos de poder das LIOs. Para as "zonas estatísticas" padrão, de três e cinco milímetros (Figura 3), o equipamento repete automaticamente os mesmos dados na janela à direita dos diferentes mapas individuais. Estes valores são, portanto, inadequados para comparações mais precisas. Por outro lado, o poder da córnea no ponto 
central de fixação não é exibido automaticamente em nenhum mapa. Quando utilizamos o recurso da "análise estatística de área" (digitando "control a"), os diâmetros centrais não são automaticamente estabelecidos, obrigando a determiná-los com a ajuda do cursor controlado pelo mouse. Apesar desta dificuldade, este recurso parece ser a forma mais correta para obter o poder da córnea numa área central, já que a variabilidade dos resultados estaria na casa do segundo ao terceiro decimal. $\mathrm{O}$ resultado pode ser impresso e inclui dados como o raio da circunferência utilizada, a área total analisada, o poder médio nessa área, seu desvio padrão e seus valores mínimo e máximo (Figura 2). Nos mapas de elevação, este recurso serve para achar o índice de Rousch (diferença entre os pontos de elevação máxima e mínima) em lugar da imprecisa localização com o cursor, na imagem exibida no monitor, dos pontos mais quente e mais frio.

Este estudo encontrou que a relação entre os poderes médios das superfícies anterior e posterior da córnea normal foi de aproximadamente 7,5/1, diferente da relação geralmente aceita de $10 / 1^{(7)}$. O menor poder positivo da superfície anterior correspondeu a um menor poder negativo da superfície posterior (Figura 4). Entretanto, aceitar esta relação, se realmente existe, seria simplificar demais a questão já que ela não pareceu ser fixa, linear ou exponencial. A correlação entre os poderes de ambas superfícies se mostrou mais razoável unicamente ao analisar áreas centrais maiores que $3 \mathrm{~mm}$ de diâmetro. Igualmente, a correlação entre os poderes de ambas superfícies da córnea com o poder derivado da sua espessura não foi boa (Tabela 3), assim como a correlação entre cada um destes três poderes com a paquimetria (Tabela 4). Estes resultados sugerem que os poderes derivados das superfícies anterior e posterior da córnea normal, de sua espessura, e a paquimetria são independentes entre si. Não sabemos ainda se nas córneas submetidas às cirurgias refrativas existe alguma correlação. Sabemos sim que estas cirurgias modificam a superfície anterior e a espessura e que poderiam também alterar indiretamente a superfície posterior da córnea ${ }^{(11-12,16)}$, e tornar, portanto, o uso do IRC impróprio nesses $\operatorname{casos}^{(1-2,7,17-18)}$.

\section{CORBSCAN}

004606

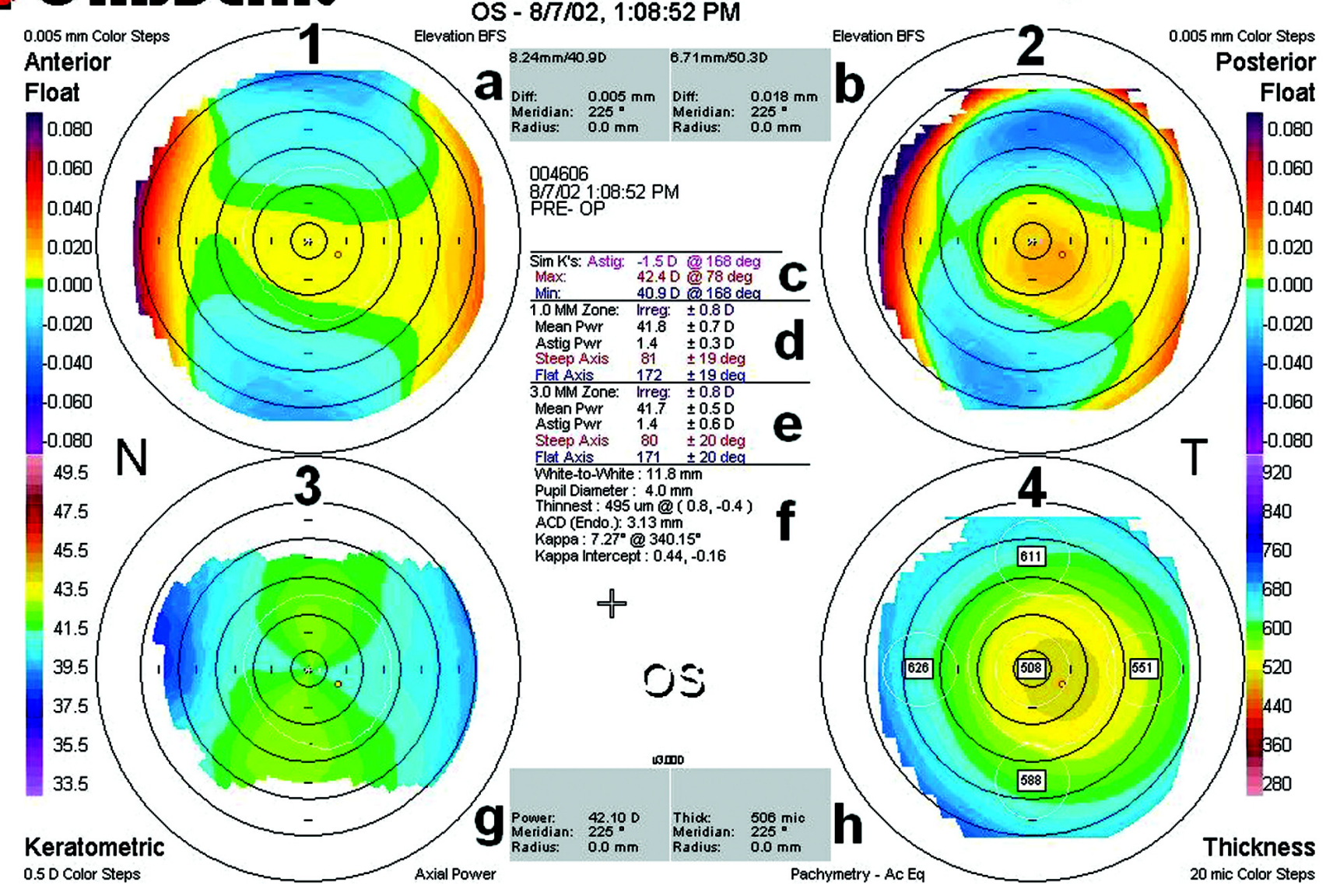

Cg Refrativa UNIFESP-EPM

Figura 3 - Exemplo da apresentação típica dos mapas ("quad map”) mostrando o mapa de elevação anterior (1), o mapa de elevação posterior (2), o mapa de poder axial ceratométrico (3), e o mapa paquimétrico (4). Na janela central superior figuram as diferenças com a melhor esfera ("best fit sphere"), no ponto indicado pelo cursor, nos mapas de elevação anterior (a) e posterior (b). Na janela central aparece o sim-K (c), a média de poder de ambos eixos (do sim-K) nas "zonas estatísticas padrão" de $1 \mathrm{~mm}$ (d) e $3 \mathrm{~mm}$ (e) de diâmetro central, o diâmetro do limbo e da pupila, a espessura mínima, a profundidade da câmara anterior e o ângulo kappa (f). Na janela central inferior aparecem o poder no mapa axial ceratométrico (g) e a espessura (h) no ponto indicado pelo cursor. Quando os mapas são exibidos individualmente no monitor, uma janela à direita exibe os dados. 


\begin{tabular}{|c|c|c|c|c|c|c|}
\hline & \multicolumn{5}{|c|}{ Diâmetro da área central analisada } & \multirow[b]{2}{*}{$\mathbf{p}$} \\
\hline & $\mathbf{n}$ & $\leq 0,02 \mathrm{~mm}$ & $1 \mathrm{~mm}$ & $3 \mathrm{~mm}$ & $5 \mathrm{~mm}$ & \\
\hline $\begin{array}{l}\text { Mapa de poder } \\
\text { óptico ceratométrico }\end{array}$ & 20 & $44,33 \mathrm{D} \pm 1,91$ & $44,40 \mathrm{D} \pm 1,84$ & $44,42 \mathrm{D} \pm 1,94$ & $44,80 \mathrm{D} \pm 2,00$ & 0,8649 \\
\hline $\begin{array}{l}\text { Mapa de poder } \\
\text { óptico total }\end{array}$ & 20 & $42,89 \mathrm{D} \pm 1,96$ & $42,97 \mathrm{D} \pm 1,91$ & $42,97 \mathrm{D} \pm 1,96$ & $43,40 \mathrm{D} \pm 1,99$ & 0,8361 \\
\hline $\begin{array}{l}\text { Mapa de poder } \\
\text { axial ceratométrico }\end{array}$ & 20 & $44,20 \mathrm{D} \pm 1,92$ & $44,17 \mathrm{D} \pm 1,92$ & $44,08 \mathrm{D} \pm 1,95$ & $43,97 \mathrm{D} \pm 1,95$ & 0,9789 \\
\hline $\begin{array}{l}\text { Mapa de poder } \\
\text { axial total }\end{array}$ & 20 & $42,62 \mathrm{D} \pm 1,91$ & $42,63 \mathrm{D} \pm 1,90$ & $42,52 \mathrm{D} \pm 1,90$ & $42,50 \mathrm{D} \pm 1,88$ & 0,9938 \\
\hline $\begin{array}{l}\text { Mapa de poder } \\
\text { tangencial total }\end{array}$ & 20 & $42,64 \mathrm{D} \pm 1,94$ & $42,63 \mathrm{D} \pm 1,90$ & $42,47 \mathrm{D} \pm 1,91$ & $42,57 \mathrm{D} \pm 1,81$ & 0,9917 \\
\hline $\begin{array}{l}\text { Mapa de poder } \\
\text { médio ceratométrico }\end{array}$ & 30 & $43,96 \mathrm{D} \pm 1,73$ & $43,92 \mathrm{D} \pm 1,74$ & $43,87 \mathrm{D} \pm 1,74$ & $43,69 \mathrm{D} \pm 1,71$ & 0,9358 \\
\hline $\begin{array}{l}\text { Mapa de poder } \\
\text { médio anterior }\end{array}$ & 30 & $48,97 \mathrm{D} \pm 1,93$ & $48,93 \mathrm{D} \pm 1,93$ & $48,88 \mathrm{D} \pm 1,94$ & $48,67 \mathrm{D} \pm 1,91$ & 0,9348 \\
\hline $\begin{array}{l}\text { Mapa de poder } \\
\text { médio posterior }\end{array}$ & 30 & $-6,62 \mathrm{D} \pm 0,36$ & $-6,62 \mathrm{D} \pm 0,34$ & $-6,56 \mathrm{D} \pm 0,28$ & $-6,35 \mathrm{D} \pm 0,28$ & $0,0030^{*}$ \\
\hline $\begin{array}{l}\text { Mapa de poder } \\
\text { médio da espessura }\end{array}$ & 30 & $0,135 \mathrm{D} \pm 0,012$ & $0,135 \mathrm{D} \pm 0,012$ & $0,136 \mathrm{D} \pm 0,012$ & $0,137 \mathrm{D} \pm 0,012$ & 0,8678 \\
\hline $\begin{array}{l}\text { Poder médio } \\
\text { da soma A/P }\end{array}$ & 30 & $42,35 \mathrm{D} \pm 1,80$ & $42,31 \mathrm{D} \pm 1,79$ & $42,32 \mathrm{D} \pm 1,75$ & $42,32 \mathrm{D} \pm 1,68$ & 0,9998 \\
\hline $\begin{array}{l}\text { Mapa de poder } \\
\text { médio total }\end{array}$ & 30 & $42,49 \mathrm{D} \pm 1,80$ & $42,45 \mathrm{D} \pm 1,79$ & $42,45 \mathrm{D} \pm 1,76$ & $42,44 \mathrm{D} \pm 1,69$ & 0,9996 \\
\hline Poder equivalente & 30 & $42,48 \mathrm{D} \pm 1,80$ & $42,44 \mathrm{D} \pm 1,79$ & $42,44 \mathrm{D} \pm 1,75$ & $42,45 \mathrm{D} \pm 1,68$ & 0,9998 \\
\hline Paquimetria & 30 & $524 \mu \pm 38,7$ & $530 \mu \pm 40,4$ & $534 \mu \pm 37,9$ & $554 \mu \pm 37,4$ & $0,0231^{*}$ \\
\hline
\end{tabular}

Neste estudo a espessura contribuiu com $+0,13 \mathrm{D}$, valor insuficiente para que o poder médio total e o poder médio da soma A/P fossem estatisticamente diferentes. Apesar de alguns autores considerarem este valor pequeno demais para merecer entrar no cálculo do poder real da córnea ${ }^{(2,4)}$, nós preferimos incluí-lo pelo nosso objetivo de eliminar as possíveis fontes de erro.

Os mapas de poder ceratométrico sempre indicaram valores maiores, entre $+0,75 \mathrm{e}+2,0 \mathrm{D}$ com quase $+1,5 \mathrm{D}$ de média,

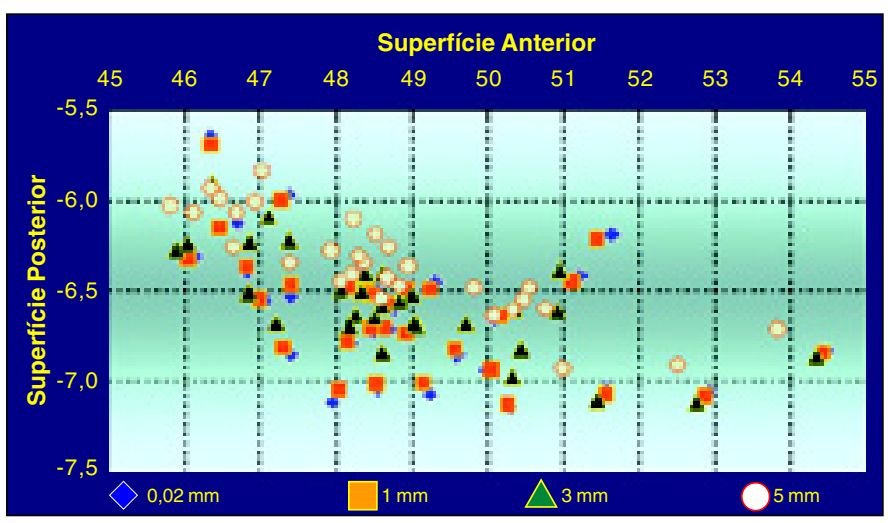

Figura 4-Relação do poder da superfície anterior com o poder da superfície posterior da córnea (em dioptrias) nos quatro diâmetros centrais analisados. que os mapas de poder total. Esta diferença foi estatisticamente significante. Nossos resultados sugerem, portanto, que $K$ é maior que $F$. Isto é, o poder da córnea normal, quando determinado pela medição do raio de curvatura da superfície anterior e o IRC, é superior (mais positivo) àquele calculado em base a todos os seus componentes ópticos (superfícies anterior e posterior, espessura e índices de refração fisiológicos). Os diversos mapas de poder ceratométrico são úteis para fins comparativos com os dados fornecidos pela ceratometria manual, computadorizada ou pela topografia com disco de Plácido. Nesse sentido, é muito provável que o $K$, em dioptrias, tradicionalmente aceito e encontrado por estes outros equipamentos também seja um valor maior que o poder total real da córnea e um valor menor que o poder real da sua superfície anterior. Parece então razoável especular que o uso do poder médio total ou do poder óptico total determinará LIOs com maior grau positivo, assim como sugerir uma revisão nas nossas práticas de aferição do poder da curva base nas lentes de contacto.

A aparição de catarata em pacientes submetidos à cirurgia refrativa é um problema que deve aumentar nos próximos anos. O sistema óptico de um olho pseudofácico tem, na córnea e nas LIOs, os mais importantes componentes ópticos que determinam a condição de emetropia. A nossa capacidade de poder mudar estas duas variáveis aumenta a nossa respon- 


\begin{tabular}{|c|c|c|c|c|c|}
\hline & $\begin{array}{l}\text { Diâmetro da } \\
\text { área central }\end{array}$ & $\begin{array}{c}\text { Média da } \\
\text { diferença } \pm \text { DP }\end{array}$ & $p$ & Intervalo & Pearson \\
\hline Poder óptico ceratométrico & $\leq 0,02 \mathrm{~mm}$ & $1,44 \pm 0,38$ & $0,0119^{*}$ & $0,70 / 2,11$ & 0,9812 \\
\hline vs & $1 \mathrm{~mm}$ & $1,42 \pm 0,46$ & $0,0107^{*}$ & $0,64 / 2,76$ & 0,9705 \\
\hline \multirow[t]{2}{*}{ Poder óptico total } & $3 \mathrm{~mm}$ & $1,44 \pm 0,30$ & $0,0123^{\star}$ & $0,80 / 2,06$ & 0,9880 \\
\hline & $5 \mathrm{~mm}$ & $1,39 \pm 0,20$ & $0,0167^{\star}$ & $0,97 / 1,67$ & 0,9952 \\
\hline Poder axial ceratométrico & $\leq 0,02 \mathrm{~mm}$ & $1,58 \pm 0,36$ & $0,0062 *$ & $0,91 / 2,14$ & 0,9826 \\
\hline vs & $1 \mathrm{~mm}$ & $1,54 \pm 0,38$ & 0,0075 * & $0,75 / 2,16$ & 0,9798 \\
\hline \multirow[t]{2}{*}{ Poder axial total } & $3 \mathrm{~mm}$ & $1,53 \pm 0,40$ & 0,0081 * & $0,31 / 2,00$ & 0,9791 \\
\hline & $5 \mathrm{~mm}$ & $1,47 \pm 0,43$ & 0,0099 * & $-0,12 / 1,84$ & 0,9756 \\
\hline Poder médio ceratométrico & $\leq 0,02 \mathrm{~mm}$ & $1,47 \pm 0,32$ & 0,0010 * & $0,76 / 2,06$ & 0,9846 \\
\hline vs & $1 \mathrm{~mm}$ & $1,47 \pm 0,29$ & 0,0010 * & $0,80 / 1,99$ & 0,9868 \\
\hline \multirow[t]{2}{*}{ Poder médio total } & $3 \mathrm{~mm}$ & $1,43 \pm 0,19$ & 0,0013 * & $1,01 / 1,75$ & 0,9942 \\
\hline & $5 \mathrm{~mm}$ & $1,25 \pm 0,13$ & 0,0030 * & $0,98 / 1,54$ & 0,9971 \\
\hline Poder médio ceratométrico & $\leq 0,02 \mathrm{~mm}$ & $1,61 \pm 0,32$ & 0,0004 * & $0,89 / 2,20$ & 0,9842 \\
\hline vs & $1 \mathrm{~mm}$ & $1,61 \pm 0,30$ & 0,0004 * & $0,95 / 2,13$ & 0,9864 \\
\hline \multirow[t]{2}{*}{ Poder médio da soma A/P } & $3 \mathrm{~mm}$ & $1,56 \pm 0,19$ & 0,0005 * & $1,15 / 1,86$ & 0,9940 \\
\hline & $5 \mathrm{~mm}$ & $1,37 \pm 0,14$ & 0,0014 * & $1,04 / 1,71$ & 0,9969 \\
\hline Poder médio total & $\leq 0,02 \mathrm{~mm}$ & $0,011 \pm 0,005$ & 0,4907 & $0,01 / 0,02$ & 1,0000 \\
\hline vs & $1 \mathrm{~mm}$ & $0,009 \pm 0,009$ & 0,4923 & $-0,02 / 0,02$ & 1,0000 \\
\hline \multirow[t]{2}{*}{ Poder equivalente } & $3 \mathrm{~mm}$ & $0,005 \pm 0,015$ & 0,4959 & $-0,04 / 0,03$ & 1,0000 \\
\hline & $5 \mathrm{~mm}$ & $0,008 \pm 0,035$ & 0,4931 & $-0,12 / 0,02$ & 0,9998 \\
\hline Poder médio total & $\leq 0,02 \mathrm{~mm}$ & $0,13 \pm 0,013$ & 0,3869 & $0,12 / 0,16$ & 1,0000 \\
\hline vs & $1 \mathrm{~mm}$ & $0,13 \pm 0,013$ & 0,3868 & $0,11 / 0,16$ & 1,0000 \\
\hline \multirow[t]{2}{*}{ Poder médio da soma A/P } & $3 \mathrm{~mm}$ & $0,13 \pm 0,019$ & 0,3882 & $0,09 / 0,17$ & 1,0000 \\
\hline & $5 \mathrm{~mm}$ & $0,12 \pm 0,037$ & 0,3946 & $0,02 / 0,17$ & 0,9998 \\
\hline
\end{tabular}

sabilidade de obter um bom resultado pós-operatório. Expressar os poderes da córnea e das LIOs nas mesmas unidades (dioptrias) não é suficiente. Os seus valores devem também representar metodologia de cálculo similar. Desde este ponto de vista, os mapas de poder óptico e os de poder médio parecem ser os mais apropriados para ser usados no cálculo das LIOs. No primeiro caso, pela similitude de princípios com as fórmulas teóricas baseadas na focalização dos raios de luz ("ray tracing"). No segundo, porque o poder médio total da córnea não exclui nenhum componente deste sistema e porque representa o seu equivalente esférico, muito usado para indicar a refração dos pacientes. A semelhança entre o poder médio total e o poder equivalente, calculado com a fórmula da lente grossa, reforça a sua escolha, entretanto, assumir este valor implica que, devido a sua forma convexo-côncava, o plano de poder efetivo da córnea está situado na frente da mesma, provavelmente tangencial a sua superfície anterior. Desconhecemos ainda o efeito que este fator possa ter, caso

\begin{tabular}{|c|c|c|c|c|}
\hline & Diâmetro da área central & Média \pm DP & Intervalo & Pearson \\
\hline & $\leq 0,02 \mathrm{~mm}$ & $7,41 \pm 0,38$ & $6,74 / 8,35$ & $-0,451$ \\
\hline Poder médio anterior & $1 \mathrm{~mm}$ & $7,41 \pm 0,35$ & $6,82 / 8,28$ & $-0,502$ \\
\hline \multirow[t]{2}{*}{$\overline{\text { Poder médio posterior }}$} & $3 \mathrm{~mm}$ & $7,46 \pm 0,23$ & $7,08 / 7,98$ & $-0,725$ \\
\hline & $5 \mathrm{~mm}$ & $7,67 \pm 0,17$ & $7,36 / 8,06$ & $-0,865$ \\
\hline & $\leq 0,02 \mathrm{~mm}$ & $366,4 \pm 28,52$ & $316 / 425$ & 0,447 \\
\hline Poder médio anterior & $1 \mathrm{~mm}$ & $365,5 \pm 28,13$ & $315 / 425$ & 0,463 \\
\hline \multirow[t]{2}{*}{ Poder médio da espessura } & $3 \mathrm{~mm}$ & $361,0 \pm 26,29$ & $312 / 419$ & 0,522 \\
\hline & $5 \mathrm{~mm}$ & $358,2 \pm 24,43$ & $306 / 409$ & 0,574 \\
\hline & $\leq 0,02 \mathrm{~mm}$ & $49,47 \pm 3,53$ & $43,5 / 57,4$ & $-0,593$ \\
\hline Poder médio posterior & $1 \mathrm{~mm}$ & $49,39 \pm 3,51$ & $43,4 / 57,3$ & $-0,596$ \\
\hline \multirow[t]{2}{*}{ Poder médio da espessura } & $3 \mathrm{~mm}$ & $48,42 \pm 3,34$ & $42,47 / 55,68$ & $-0,623$ \\
\hline & $5 \mathrm{~mm}$ & $46,72 \pm 3,02$ & $41,28 / 53,13$ & $-0,665$ \\
\hline
\end{tabular}




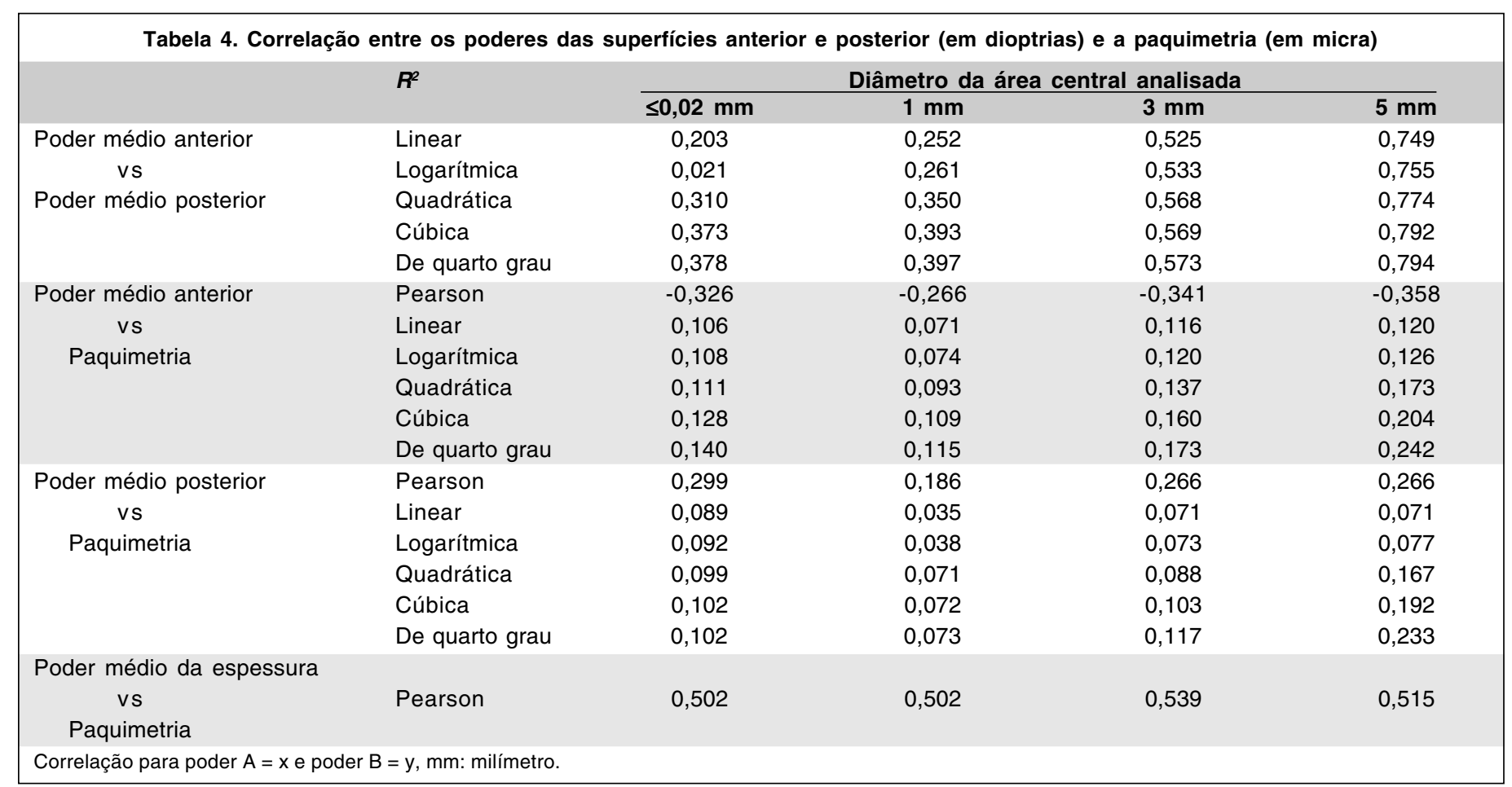

for incluído, no cálculo do poder das LIOs, nas fórmulas que usam a profundidade da câmara anterior, isto é, a distância entre a superfície posterior da córnea e a anterior do cristalino. Os mapas de elevação são, talvez, o grande aporte inovador do Orbscan-II na topografia da córnea. Entretanto, eles e os mapas de poder axial, tangencial e astigmático não parecem coincidir com os nossos objetivos.

A variação da espessura média da córnea central, embora aparentemente pequena, foi estatisticamente significante. Desconhecemos se este aumento de quase $30 \mathrm{~mm}$, entre o centro e os $5 \mathrm{~mm}$ centrais, reflete também uma maior variabilidade das medidas pelo maior número de pontos analisados pelo equipamento.

A capacidade do Orbscan-II de analisar a região central da córnea parece ter sido pouco pesquisada de modo sistemático e pode ser um passo importante no cálculo do poder das LIOs. Enquanto a ceratometria manual mede uma área variável pouco maior em córneas mais planas ${ }^{(19)}$, a topografia convencional com disco de Plácido infere o centro da córnea, sem medílo, a partir de anéis para-centrais ${ }^{(20)}$. Este estudo determinou que o poder total da córnea normal é o mesmo, independente do tamanho da área central analisada, desde o centro exato até os $5 \mathrm{~mm}$ de diâmetro. $\mathrm{O}$ único componente que variou foi o poder negativo da superfície posterior que diminuiu conforme aumentou a área. Não sabemos se o mesmo acontece em córneas com ceratocone ou após as diversas cirurgias refrativas, onde o centro é a região mais alterada da córnea. Por enquanto, para o cálculo das LIOs, não parece tão razoável usar o poder do centro exato da córnea, como tem sido sugerido por outros autores ${ }^{(1)}$, mas usar o poder de áreas centrais com diâmetro entre $1 \mathrm{e} 4 \mathrm{~mm}$, considerando que a fóvea mede $1,5 \mathrm{~mm}^{(21)}$ e que estes diâmetros, centralizados no eixo da visão, estariam incluindo o ápice e o centro geométrico da córnea, geralmente com $5^{\circ}$ a $10^{\circ}$ de separação do eixo visual. $\mathrm{A}$ área de $3 \mathrm{~mm}$ corresponde aproximadamente à área pupilar delimitada no Orbscan-II e à área central livre freqüentemente encontrada após ceratotomia radial, e poderia ser usada para fins comparativos com outros métodos de medição que usam uma área de exame parecida. Isto é, a ceratometria convencional $(2,6 \mathrm{~mm}$ no ceratómetro AO e 3,1 mm no ceratómetro $\mathrm{B} \& \mathrm{~L})$ e a ceratometria central simulada $(\operatorname{sim}-K)^{(19-20)}$. O valor médio de uma área maior que $4 \mathrm{~mm}$ certamente poderia estar introduzindo fatores adicionais de erro de cálculo em córneas com astigmatismo irregular, ceratocone ou cirurgia refrativa.

Estudos adicionais já estão sendo realizados a fim de se estabelecer conclusões mais precisas que ajudem a evitar os erros observados no cálculo do poder das LIOs, principalmente nos pacientes com cirurgia refrativa prévia ${ }^{(1-2,7,17-18)}$.

\section{CONCLUSÕES}

O poder da córnea, determinado tradicionalmente pela medição da curvatura da superfície anterior e o índice de refração ceratométrico (IRC) da córnea, é maior que o poder total real da córnea central, calculado em base às curvaturas das superfícies anterior e posterior, à espessura e os índices de refração fisiológicos, $(K>F)$. Enquanto o poder da superfície anterior e o poder total da córnea normal não mudam, o poder da superfí- 
cie posterior e a paquimetria variam segundo o tamanho da área central estudada. $\mathrm{O}$ poder da superfície anterior foi, em média, entre 7,4 e 7,7 vezes maior que o poder da superfície posterior. Entretanto, quando a área analisada foi menor que 5 mm de diâmetro, a correlação entre ambos não pareceu ser fixa, linear, quadrática, cúbica, de quarto grau nem logarítmica. A falta de uma boa correlação sugere que os três componentes do poder total da córnea e a paquimetria são independentes entre si.

\section{ABSTRACT}

Purpose: To evaluate the dioptric power of the normal central cornea. Methods: Retrospective analysis of combined slitscanning and Placido-ring topography in 30 normal eyes. Results: Keratometric-mean, anterior-mean, thickness-mean, and total-mean powers; keratometric-optical and total-optical, keratometric-axial and total-axial, and total-tangential powers; equivalent power and the addition of anterior and posterior mean powers, were similar $(p \geq 0.8361)$ in the four analyzed central areas with $0.04,1.0,3.0$, and $5.0 \mathrm{~mm}$ diameter. The posterior-mean power decreased $(p=0.0030)$ from $-6.62 \mathrm{D}$ to $-6.35 \mathrm{D}$. The average thickness increased $(p=0.0231)$ from $524 \mu \mathrm{m}$ to $554 \mu \mathrm{m}$, representing $0.13 \mathrm{D}$ of total-mean corneal power. Keratometric-optical $(p \leq 0.0167)$, keratometric-axial $(p \leq 0.0099)$, and keratometric-mean powers $(p \leq 0.0030)$ were approximately $1.5 \mathrm{D}$ more positive than the respective total powers. Total-mean power was equal to equivalent power $(p \geq 0.4907)$, and to the addition of anterior-mean and posteriormean powers $(p \geq 0.3868)$. Anterior-mean power was about 7.5 greater than posterior-mean power. Conclusions: The real power of the cornea calculated using both surfaces, its thickness, and the physiologic refraction indices is smaller (less positive) than the power usually accepted and determined by the anterior corneal surface radius and the keratometric index of refraction $(F<K)$. Anterior-mean/posterior-mean power ratio is not ten-to-one as conventionally assumed. Power of posterior surface and thickness vary according to the size of the analyzed area. However, power of anterior surface and total power of the cornea remain the same. Components of total corneal power and pachymetry seem to be independent of each other.
Keywords: Cornea/physiology, Corneal topography/statistics \& numerical data, Ocular refraction, Intraocular lenses

\section{REFERÊNCIAS}

1. Holladay JT. Intraocular lens power calculations for the refractive surgeon. Oper Tech Cataract Refract Surg 1998;1:105-17.

2. Seitz B, Langenbucher A. Intraocular lens calculations status after corneal refractive surgery. Curr Opin Ophthalmol 2000;11:35-46.

3. Holladay JT, Maverick KJ. Relationship of actual thick intraocular lens optic to the thin lens equivalent. Am J Ophthalmol, 1998;126:339-47.

4. Speicher L. Intra-ocular lens calculation status after corneal refractive surgery. Curr Opin Ophthalmol 2001;12:17-29.

5. Javal E. Memories d'ophtalmométrie. Paris, França: Libraire de L'Académie de Médicine; 1891.

6. Schor P, Carvalho LA. Introdução e histórico da topografia da córnea. In Polisuk P. Topografia da córnea. Atlas clínico. Rio de Janeiro: Cultura Médica; 2000. Cap.1.

7. Seitz B, Langenbucher A. Intraocular lens power calculation in eyes after corneal refractive surgery. J Refract Surg 2000;16:349-61.

8. Marsich MM, Bullimore MA. The repeatability of corneal thickness measures. Cornea 2000;19:792-5.

9. Naroo SA, Charman N. Changes in posterior corneal curvature after photorefractive keratectomy. J Cataract Refract Surg 2000;26:872-8.

10. Chaves HV. Topografia por varredura com iluminação de fenda. In: Polisuk P. Topografia da córnea. Atlas clínico. Rio de Janeiro: Cultura Médica; 2000. Cap. 12 .

11. Auffarth GU, Wang L, Völcker HE. Keratoconus evaluation using the Orbscan Topography System. J Cataract Refract Surg 2000;26:222-8.

12. Seitz B, Torres F, Langenbucher A, Behrens A, Suarez E. Posterior corneal curvature changes after myopic laser in situ keratomileusis. Ophthalmology 2001;108:666-72; discussion p.673.

13. Yaylali V, Kaufman SC, Thompson HW. Corneal thickness measurements with the Orbscan Topography System and ultrasonic pachymetry. J Cataract Refract Surg 1997;23:1345-50.

14. Liu Z, Huang AJ, Pflugfelder SC. Evaluation of corneal thickness and topography in normal eyes using the Orbscan corneal topography system. Br J Ophthalmol 1999;83:774-8.

15. Michaels DD. Lens optics. In: Michaels DD, Visual optics and refraction: a clinical approach. Saint Louis: Mosby; 1975. Cap.3.

16. Kamiya K, Oshika T, Amano S, Takahashi T, Tokunaga T, Miyata K. Influence of excimer laser photorefractive keratectomy on the posterior corneal surface. J Cataract Refract Surg 2000;26:867-71.

17. Seitz B, Langenbucher A, Nguyen NX, Kus MM, Küchle M. Underestimation of intraocular lens power for cataract surgery after myopic photorefractive keratectomy. Ophthalmology 1999;106:693-702.

18. MacEwan JR, Massengil RK, Friedel SD. Effect of keratometer and axial length measurement errors on primary implant power calculations J. Cataract Refract Surg 1990;16:61-70,

19. Keeney AH. The cornea and the sclera. In: Keeney AH, Ocular examination, basis and technique. Saint Louis: Mosby; 1970. p.73-91.

20. Polisuk P. Compreendendo a topografia da córnea. In: Polisuk P. Topografia da córnea. Atlas clínico. Rio de Janeiro: Cultura Médica; 2000. Cap.4.

21. Kozart DM. Anatomic correlates of the retina. In: Duane TD, editor. Clinical Ophthalmology. Philadelphia: Harper \& Row; 1981. Vol.3,Cap.1.

Nos artigos enviados para publicação, o nome dos autores

e suas afiliações devem estar completos. Isso facilitará a indexação e os links com as bases de dados e o CV Lates. 